\title{
Phenytoin Interaction with Enteral Feedings Administered through Nasogastric Tubes
}

\author{
David Fleisher, Ph.D., Neel Sheth, and Jim H. Kou, Ph.D. \\ From the College of Pharmacy, University of Michigan, Ann Arbor, Michigan
}

\begin{abstract}
Inadequate drug plasma levels have been associated with the administration of phenytoin with enteral feedings through nasogastric (NG) tubes. It is demonstrated in this study that loss of phenytoin to tubing is a function of $\mathrm{pH}$. Nonionized phenytoin is irreversibly bound to NG tubing from solution at the $\mathrm{pH}$ of enteral nutrient solutions while this is not the case for anionic phenytoin in unbuffered water or saline. In experiments pulsing phenytoin through glass $v s$ NG tubing perfused with buffer at varying $\mathrm{pH}$, reversible loss to tubing was observed at high $\mathrm{pH}$ while irreversible loss was observed
\end{abstract}

at low $\mathrm{pH}$. In addition, the irreversible loss of phenvtoin was greater in NG tubing than glass particularly at low pH. It is suggested that in those cases where tubing is placed into the duodenum, inadequate gastrointestinal residence time for dissolution of phenytoin solid and suspension dosage forms coupled with irreversible drug loss from solution to NG tubing will result in decreased phenytoin absorption and subsequently lower drug plasma levels. (Journal of Parenteral and Enteral Nutrition 14:513-516, 1990)
Numerous reports describing an interaction with nasogastric (NG) enteral feedings and the antiepileptic phenytoin (PHT) have appeared in the literature since 1982. These reports include medical case studies as well as in vivo and in vitro investigations designed to elucidate the mechanism of this interaction. The case studies reported clinical effects as a result of decreased PHT plamsa levels in the presence of enteral NG feedings ${ }^{1,2}$ and increased levels when feedings were discontinued. ${ }^{3,4}$ Because of the narrow therapeutic index of PHT, under or overdosage results in more serious (and thus reportable) clinical events than are observed for other drugs. ${ }^{5}$ Recommendations for PHT administration with respect to dosage and timing during enteral NG feeding schedules remain unsettled. ${ }^{1-4}$

In vitro studies have focused on $\mathrm{PHT}$ loss to $\mathrm{NG}$ tubing ${ }^{3,6}$ and loss to enteral feeding components. ${ }^{7.8}$ In this regard, binding to caseinate salt components of both enteral feedings ${ }^{7}$ and dairy products ${ }^{9}$ has been implicated while PHT loss to NG tubing from drug in suspension is only significant when tubing irrigation is not performed after PHT administration. ${ }^{6}$ Interestingly, this latter study suggested that greater loss to tubing occurred from diluted as opposed to undiluted PHT suspensions. ${ }^{6}$

In vivo studies in patients and normal subjects have focused on the influence of oral (without NG tubing) administration of enteral feedings on PHT bioavailability from Dilantin suspensions ${ }^{10}$ and capsules. ${ }^{11}$ Dilantin (Parke Davis/Warner Lambert brand of PHT) suspension contains PHT free acid while the capsules contain the sodium salt of PHT. Both studies failed to show significant effects of these feedings on PHT bioavailability as compared to controls over $80 \mathrm{hr}^{10}$ and $48 \mathrm{hr} .{ }^{11}$ However, it is our observation that the data in both

\footnotetext{
Received for publication, July 5, 1989

Accepted for publication. January 9, 1990.

Reprint requests: David Fleisher, $\mathrm{PhD}$, College of Pharmacy, Lniversity of Michigan, Ann Arbor, MI 48109-1065.
}

studies show higher PHT plasma levels up to $10 \mathrm{hr}$ in the presence of enteral feedings us controls.

The results of this report were obtained following studies in which nutrient effects on gastrointestinal (GI) transit time in vivo ${ }^{12}$ and on PHT intestinal membrane permeability in situ ${ }^{13}$ were observed. Delay of stomach emptying in the fed-state, as monitored by radiotelemetry, correlated with higher PH'T plasma levels from orally administered PHT capsules and suspensions in beagle dogs. ${ }^{12}$ These results are consistent with the early time data in human studies reported previously ${ }^{10,11}$ and suggest that if the dosage form is retained in the stomach, greater GI residence time is available for dissolution of PH'T free acid.

In situ rat intestinal perfusions are performed in our laboratories using glass or Teflon tubing as inlet and outlet conduits. To measure intestinal drug uptake from perfusion solution, drug loss to tubing under the perfusion conditions is routinely evaluated in a control experiment. In the case of PHT, minimal drug loss to glass and Teflon tubing is observed in jejunal perfusions carried out at $\mathrm{pH} 6.5$ in which the flow rate is $0.5 \mathrm{ml} / \mathrm{min}$ over $50-100 \mathrm{~cm}$ inlet tubing lengths. ${ }^{13}$ The observation that greater PHT loss to NG tubing occurs from diluted Dilantin suspensions ${ }^{6}$ prompted experimental measurement of PHT loss to NG tubing as a function of drug input and perfusion $\mathrm{pH}$.

\section{MATERIALS AND METHODS}

Solutions of PHT free acid $80 \mu \mathrm{M}$ (intrinsic aqueous solubility $83 \mu \mathrm{M}$ as determined in phosphate buffer at $\mathrm{pH} 5$ and $\mathrm{pH}$ 3) were prepared and traced with 5,5diphenyl- $\left[4-{ }^{14} \mathrm{C}\right]$ hydantoin (Amersham International. 58 $\mathrm{mCi} / \mathrm{nmol}$ ) at $5.93 \times 10^{\mathrm{n}} \mathrm{dpm} / \mathrm{ml}$. Phosphate buffer at $65 \mathrm{mM}$ was made up at $\mathrm{pH} 3,7$. and 12 and perfused through $40 \mathrm{~cm}$ of both glass and $\mathrm{NG}$ tubing (Entriflex distributed by Biosearch Medical Productsi at $11.5 \mathrm{ml}$ / min. Entriflex tubing is made of polyurethane and is 
lubricated on the interior and exterior surfaces with Hydromer [polyvinylpyrrolidone (PVP), Hydromer Inc] for insertion and removal of a flow-through stylet activated by injecting water to ease the passage of the tubing. During the phosphate buffer perfusions, PHT solutions were pulsed over a $30-\mathrm{sec}$ time interval $\left(t_{p}\right.$, Table I) and the time course of $\left[{ }^{14} \mathrm{C}\right]$ phenytoin output levels were monitored by liquid scintillation counting. An additional experiment was carried out in which perfusion of the enteral nutrient solution, Osmolite HN (Ross Laboratories, pH 6.4), replaced the phosphate buffer and PHT solution was pulsed as before.

\section{RESULTS}

Plots comparing PHT output from glass tubing os NG tubing over $10-20 \mathrm{~min}$ are shown in Figure 1 for the various perfusion solutions. In all cases, greater loss of PHT to NG tubing was observed as compared with drug loss to glass tubing (Table I, Fig. 1). Standard errors of the mean are not given in the data presentation. However, duplicate experiments were performed which yielded equivalent results. The form of the data in these plots can be compared with an ideal solute residence time distribution consistent with laminar flow of solvent fluid through the cylindrical tubing. ${ }^{14}$ Deviations from the ideal flow pattern are suggestive of either solvent flow perturbations or solute interaction with the wall of the tubing. The positioning of the tubing as well as its length and flow rate used in these studies represent experimental conditions which minimize deviations from an ideal laminar flow pattern. As a result, deviations from the ideal solute residence time pattern (as generated by laminar flow) are indicative of the type of interaction occurring between the drug and the tubing wall, whether reversible or irreversible.

PHT is a weak acid ( $\mathrm{pKa}=8.1$ ) and the data in Figure 1 suggest that PHT anion binds reversibly to the NG tubing while the uncharged free acid is removed in an irreversible fashion. In particular, at pH 12 a greater degree of tailing in the data is indicative of initial binding of anion (the drug is totally ionized at this $\mathrm{pH}$ ) while little PHT mass is lost to the tubing (Table I) over the total time course of the experiment. This suggests that the binding of the anion is reversible because the drug eventually exits the tubing as perfusion continues. The data at $\mathrm{pH} 3$ (in which the drug is totally nonionized) does not show the tailing characteristic of reversible binding (Fig. 1A) and in addition shows substantial loss of PHT mass to NG tubing (Table I). This latter result is suggestive of irreversible loss of nonionized PHT to the NG tubing.

Although administration of PHT at pH 3 and 12 does

TABLE I

Total recovered mass (\%) over 20 min in phosphate buffer $\left({ }^{14} \mathrm{C}\right.$ traced $80 \mu M$ PHT; $\left.t_{p}=30 \mathrm{sec}\right)$

\begin{tabular}{lcccc}
\hline Tubing & $\mathrm{pH} 3$ & $\mathrm{pH} 7^{*}$ & $\mathrm{pH} 12$ & $\begin{array}{c}\mathrm{pH} 12 \text { drug; } \\
\mathrm{pH} \mathrm{6.4} \mathrm{Osmolite}\end{array}$ \\
\hline Glass & 99.8 & 80.9 & 98.8 & 86.1 \\
NG & 38.7 & 40.6 & 88.9 & 63.0
\end{tabular}

${ }^{*} \mathrm{pH} \tau$ experiment was only carried out for $12 \mathrm{~min}$ with a $t_{p}=20$ sec. not reflect realistic clinical situations, it does represent the extremes at which this drug would be presented to the tubing in totally nonionized or ionized form, respectively. The goal of this project was to separate PHT loss from solution from events associated with dissolution of solid PH'T in capsule and suspension dosage forms. It would be expected, for example, that if Dilantin Injectable $(\mathrm{pH}=12)$ were administered through NG tubing in Osmolite $(\mathrm{pH}=6.4$ ) feedings, that precipitation of the free $\operatorname{acid}^{15}$ would remove significantly more drug from availability for absorption than removal by the NG tubing. In this study, however, PHT was pulsed below its intrinsic aqueous solubility (all the drug is in solution) and loss of PHT in Osmolite to tubing (Fig. 1D) follows a pattern similar to that seen in phosphate buffer at $\mathrm{pH}$ 7 (Fig. 1C).

\section{DISCUSSION}

Case studies in which PH'T is administered by NG tubing through which enteral feedings are given have frequently resulted in depressed PHT plasma levels such that the clinical consequences of effective underdosage are observed. ${ }^{1-4}$ In vitro studies have demonstrated significant loss of PHT when mixed with enteral nutrient solutions, ${ }^{7,8}$ yet when PHT is administered orally with enteral nutrient solutions without NG tubing, PHT bioavailability is not compromised. ${ }^{10,11}$

In a study performed in beagle dogs, ${ }^{12}$ PHT capsules were administered orally and stomach emptying was monitored by radiotelemetry (Heidelberg capsule, Electro-Medical Devices). ${ }^{16}$ The study demonstrated that fedstate delay of stomach emptying resulted in a longer GI residence time over which $\mathrm{PHT}$ dissolution could occur. [The fasted state stomach emptying pattern is dictated by the cyclic nature of the migrating motor complex (MMC)]. PHT plasma levels were seen to correlate with the length of time between oral dosage and stomach emptying. In one of the dogs, stomach emptying occurred shortly after the PHT capsule was administered. Although equal fluid volumes $(150 \mathrm{ml}$ water) were given with the PHT capsule orally, drug plasma levels over 8 hr were 3-5 times lower in this dog. It is conjectured that much of the undissolved, hydrophobic PH'T does not empty continuously from the stomach with the fluid because it tends to aggregate as large particles ${ }^{17}$ which empty undissolved with the MMC.

In comatose patients, stomach emptying is compromised and NG feeding tubes are frequently positioned to empty into the duodenum. ${ }^{18}$ It is hypothesized that this bypassing of the stomach followed by continuous enteral feeding dictates inadequate time for PHT dissolution in the GI tract and may contribute to the low PHT levels observed in the case studies. ${ }^{1-4}$

Furthermore, the in vitro studies reported here show that a substantial amount of dissolved PHT is removed by NG tubing. The Hydromer coating used to lubricate the tubing interior and exterior surfaces is PVP. PHTPVP coprecipitates have been reported to markedly enhance PHT aqueous dissolution rates and subsequent GI absorption. Supersaturation 2-3 times the aqueous PHT solubility were maintained for $2 \mathrm{hr} .{ }^{19}$ Structural similar- 

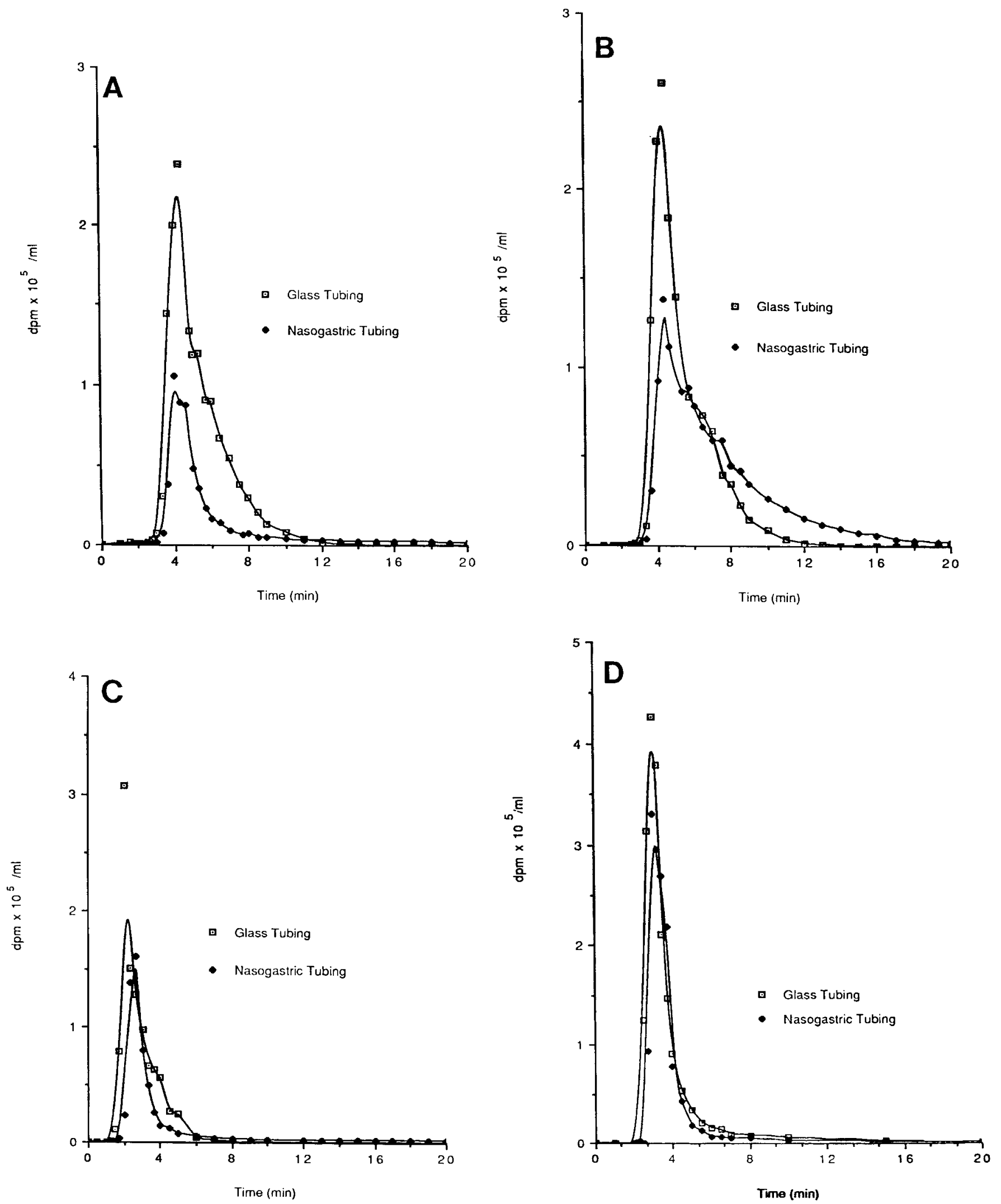

Fig. 1. Recovery from pulsed $\left[{ }^{1+} \mathrm{C}\right] \mathrm{PHT}$ in phosphate buffer at $\mathrm{pH}=3(A), 12(B)$. and $T(C)$ and in Osmolite $\mathrm{HN}$ at $\left.\mathrm{pH}=f, t, I\right)$, through glass and NG tubing. 
ities between PVP and PHT suggest that the Hydromer coating may be responsible for extracting nonionized PHT from solution.

When a PHT suspension is administered by NG tubing, short-term PHT blood levels may be decreased by loss of dissolved PH'T to the tubing and binding of PHT to caseinate salt components of enteral feeding solutions. ${ }^{\top}$ Long-term PHT availability for absorption may be reduced in those cases where undissolved PHT is not held up in the stomach. When the tubing empties directly into the small intestine and PHT is followed by continuous feedings, GI residence time may not be adequate for total PHT dissolution and overall PHT bioavailability will be correspondingly compromised.

Previous in vitro studies have demonstrated negligible loss of PHT to NG tubing. ${ }^{3,6}$ These studies were performed with suspensions and not solutions. As a result the drug lost to tubing from solution is negligible when compared with the total drug potentially available from undissolved drug in the suspension. This study demonstrates that drug irreversibly lost from solution to NG tubing is substantial if the $\mathrm{pH}$ is low enough for the nonionized form to dominate. Furthermore, if the stomach empties immediately or tubing is positioned to empty into the duodenum, solid PHT will not be provided with adequate GI residence time for complete dissolution. Lastly, the in vitro data presented here are consistent with the unusual finding of a previous study ${ }^{6}$ showing greater loss of PHT to NG tubing from diluted Dilantin suspensions than from undiluted suspensions.

If it is necessary to administer PHT by NG tube, it is recommended that Dilantin capsules be given and that the tubing be flushed with unbuffered solution (because unbuffered sodium PHT dissolution results in high solution $\mathrm{pH}$, reducing irreversible drug loss to the tubing). If the tubing empties into the duodenum, GI residence time is compromised, providing less time for PHT dissolution. Variable absorption, in this latter situation, will certainly be a function of both PHT dosage form and choice of enteral nutrition. ${ }^{18}$ PHT plasma levels should be monitored in either situation because decreased absorption may couple with other contributions to drug plasma level variability. Monitoring of plasma levels is particularly important in the face of this variability because of PHT's narrow therapeutic index.

\section{ACKNOWLEDGMENTS}

This research was supported by University of Michigan Rackham Graduate School Grant 386043 and $\mathrm{Na}$ tional Institutes of Health Grant 1 R29 NS24616-02.

\section{REFERENCES}

1. Bauer LA: Interference of oral phenytoin absorption by continuous nasogastric feedings. Neurology 32:570-572, 1982

2. Hatton RC: Dietary interaction with phenytoin. Clin Pharm 3:110111,1984

3. Ozuna J, Friel P: Effect of enteral tube feeding on serum phenytoin levels. J Neurosurg Nurs 16:289-291, 1984

4. Saklad JJ, Graves RH, Sharp WP: Interaction of oral phenytoin with enteral feedings. JPEN 10:322-323, 1986

5. Longe RL, Smith OB: Phenytoin interaction with an oral feeding results in loss of seizure control. J Am Geront Soc 36:542-544, 1988

6. Cacek T, DeVito JM, Koonce JR: In vitro evaluation of nasogastric administration methods for phenytoin. Am J Hosp Pharm 43:689692,1986

7. Hooks MA, Longe RL, Taylor AT: Recovery of phenytoin from an enteral nutrient feeding. Am J Hosp Pharm 43:685-688, 1986

8. Smith $\mathrm{OB}$, Longe RL, Altman RE, et al: Recovery of phenytoin from solutions of caseinate salts and calcium chloride. Am J Hosp Pharm 45:365-368, 1988

9. Jann MW, Bean J, Fidone GS: Interaction of dietary pudding with phenytoin. Pediatrics 78:952-953, 1986

10. Krueger KA, Garnett WR, Comstock TJ, et al: Effect of two administration schedules of an enteral nutrient formula on phenytoin bioavailability. Epilepsia 28:706-711, 1987

11. Nishimura LY, Armstrong EP, Plezia PM, et al: Influence of enteral feedings on phenytoin sodium absorption from capsules. Drug Intell Clin Pharm 22:130-133, 1988

12. Fleisher D, Lippert CL, Sheth N, et al: Nutrient effects on gastrointestinal drug absorption. J Controlled Rel 11:41-49, 1990

13. Fleisher D, Sheth N, Griffin H, et al: Nutrient influences on rat intestinal phenytoin uptake. Pharm Res 6:332-337, 1989

14. Amidon GL, Kou J, Elliot RL, et al. Analysis of models for determining intestinal wall permeabilities. J Pharm Sci 69:13691373,1980

15. Pfeifle CE, Adler DS, Gannaway WL: Phenytoin sodium solubility in intravenous solutions. Am $J$ Hosp Pharm 38:358-362, 1981

16. Dressman JB, Amidon GL: Radiotelemetric method for evaluating enteric coatings in vivo. J Pharm Sci 73:935-938, 1984

17. Arnold K, Gerber N, Levy G: Absorption and dissolution studies on sodium diphenylhydantoin capsules. Can J Pharm Sci 5:89-95, 1970

18. Maynard GA, Jones KM, Guidry JR: Phenytoin absorption from tube feedings. Arch Intern Med 147:1821, 1987

19. Seikawa H, Fujiwara J, Naganuma T, et al: Dissolution behavior and gastrointestinal absorption of phenytoin in phenytoin-polyvinylpyrrolidone coprecipitate. Chem Pharm Bull 26:3033-3039, 1976 